\title{
A importância da prática regular de exercícios físicos para manutenção do sono e memória em mulheres donas de casa acima dos 50 anos de idade
}

14

\author{
The importance of physical exercise of \\ regular practice for sleep maintenance \\ and memory in housewives women house \\ above 50 years of age
}

FisiSenectus . Unochapecó Ano 4, n. 2 - Jul/Dez. 2016 p. $30-37$

João Paulo Alves do Couto. jcouto@unece.br

Fisioterapeuta. Doutor em Engenharia Biomédica pela Universidade Camilo Castelo Branco, Brasil. Faculdades Integradas do Extremo Sul da Bahia - UNESULBAHIA.

Simone Giovaneli Camata. simonelgcamata@outlook.com Graduação em Fisioterapia. Centro Universitário do Espírito Santo/ES.

\section{Resumo}

Introdução: 0 processo de envelhecimento fisiológico não ocorre de forma igual no corpo humano, cada sistema responde de forma particular ao envelhecimento. 0 sistema nervoso central é o que sofre maiores alterações, sendo que o declínio da capacidade cognitiva, da memória e também do ciclo sono-vigília são os principais fatores alterados. Objetivo: avaliar a qualidade do sono e memória de todas as participantes pela aplicação do Miniexame do Estado Mental e Escala de sono de Epworth e comparar quais grupos apresentam melhor capacidade de memória e qualidade de sono. Materiais e métodos: Foi realizado o estudo com 60 mulheres, divididas em 3 grupos de 20 pessoas: grupo sedentárias, grupo praticantes de atividade física há um ano e grupo praticantes de atividade física há mais de dois anos. Os participantes tinham idade entre a 50 e 75 anos, com escolaridade igual ou inferior a quarta série do Ensino Fundamental. Resultados: melhores desempenhos tanto no teste Minimental como na escala de Epworth das participantes ativas em relação às sedentárias e também melhores resultados entre as participantes que realizam atividade física há mais de dois anos em relação às que praticam há apenas um ano. Conclusão: o estudo indica os efeitos benéficos do exercício físico sobre a melhora da qualidade de sono, memória e qualidade de vida.

\section{Palavras-chave}

Memória; Sono; Atividade física. 


\begin{abstract}
Introduction: The process of physiological aging does not occur equally in the human body, each system responds in a particular way to aging. The central nervous system is the one that undergoes major alterations, being that the decline of the cognitive capacity, of memory and also of Sleep-Wake Cycle are the main altered factors. Objective: to evaluate the sleep and memory quality of all participants by applying the Minimental State Examination and Epworth Sleep Scale test and compare which groups present better memory capacity and sleep quality. Materials and methods: The study consisted of 60 women, divided into 3 groups of 20 people: the sedentary group, the physical activity group for one year and the physical activity group for more than two years. Participants were aged between 50 and 75 years old, with schooling equal to or less than the fourth grade of elementary school. Results: better performance in both the MinimentalState Examination and Epworth scores of the active participants compared to the sedentary ones, and also better results among the participants who performed physical activity more than two years compared to those who practiced only one year ago. Conclusion: the study indicates the beneficial effects of physical exercise on the improvement of sleep quality, memory and quality of life.
\end{abstract}

\title{
Keywords
}

Memory; Sleep; Physical activity.

\section{Introdução}

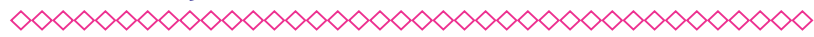

A população mundial hoje apresenta um perfil epidemiológico caracterizado por grande número de pessoas com mais de 60 anos. A tendência é que essa massa representante da terceira idade aumente consideravelmente em algumas décadas. Essa mudança no aspecto demográfico se deve ao avanço tecnológico nas áreas médicas, à mudança dos hábitos de vida da população em geral e a um decréscimo significativo na taxa de natalidade e mortalidade em todo o mundo ${ }^{1}$.

Com o aumento da expectativa de vida, alguns fatores devem ser analisados, como o surgimento de alterações e patologias relacionadas à idade avançada. 0 envelhecimento se apresenta de diferentes formas para cada indivíduo ao longo da vida, já que é um processo heterogêneo. Esse processo pode vir acompanhado de alterações no padrão de sono habitual, o que costuma ser a uma queixa comum dos idosos².

As modificações no padrão de sono do idoso causam repercussões na função psicológica, no sistema imunológico, no desempenho, na resposta comportamental, no humor e na habilidade de adaptação. São frequentes as queixas referentes à menor duração do sono noturno, ao despertar precoce pela manhã, à dificuldade para iniciar e/ ou manter o sono, sonolência diurna, aumento dos cochilos, comprometimento cognitivo e memória ${ }^{3}$.

Nesse ponto de vista, a preservação do estado cognitivo principalmente da memória e uma boa regulação dos ciclos fisiológicos, como o ciclo sono-vigília, são muito importantes para se manter um bom grau de independência e uma maior funcionalidade em um aspecto geral ${ }^{4}$.

0 objetivo deste estudo é ressaltar a importância da prática regular de exercícios físicos para que haja uma manutenção de forma adequada da memória e do sono, fatores estes que são fundamentais para garantir o bem-estar dos indivíduos na terceira idade. Além dessa constatação, objetiva-se informar os leitores acerca da necessidade de manter-se ativo desde a juventude até a terceira idade.

\section{Materiais e métodos}

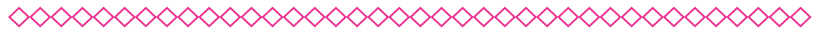

Esta pesquisa foi aprovada pelo Comitê de Ética em Pesquisa do Centro Universitário do Espírito Santo (UNESC/ES), parecer $\mathrm{n}^{0} 220.608$, pelo protocolo CAAE 35924114.8.0000.5062. 


\section{Modelo experimental}

Realizou-se um estudo quantitativo e qualitativo com um grupo composto de 60 mulheres com idade entre 50 a 75 anos, donas de casa, residentes na cidade de Marilândia/ES, sedentárias e ativas, com escolaridade igual ou inferior à quarta série do Ensino Fundamental.

As participantes que se exercitavam fazem parte do grupo denominado "Idade Ativa", composto por idosos de ambos os sexos que se reúnem diariamente no centro de convivência do idoso "Júlia Bragatto Camata", na cidade de Marilândia, sob a orientação de uma educadora física, e que realizam ginástica aeróbica e caminhada. E algumas delas praticavam as duas modalidades e outras apenas a ginástica aeróbica.

Todas as participantes foram devidamente esclarecidas sobre o objetivo da pesquisa, e cada uma recebeu um termo de consentimento $E$, após a leitura, assinaram o referido termo, dando autorização para o uso e a publicação dos dados coletados.

Com intuito de não interferir nos resultados do estudo, foi adotado, como critério de exclusão, mulheres com qualquer quadro de demência diagnosticado por médico neurologista ou psiquiátrico, indivíduos em uso de medicações antidepressivas ou qualquer tipo de sonífero ou sedativo e mulheres com escolaridade superior a quarta série do Ensino Fundamental e residentes em outro município que não seja o de Marilândia.

\section{Procedimento experimental}

A pesquisa foi realizada nas dependências do Centro de Convivência do Idoso "Júlia Bragato Camata", na cidade de Marilândia, de forma individual, onde cada participante tinha o seu horário agendado, sempre no período da manhã entre as $8 \mathrm{~h}$ e $10 \mathrm{~h} 30 \mathrm{~min}$.

A pesquisa efetivou-se por meio da aplicação do Miniexame do Estado Mental, para avaliação da memória ${ }^{5}$. Também foi aplicada a Escala de Epworth, para avaliação do índice de sonolência diurna das participantes do estudo ${ }^{6}$.

Ao chegar ao local agendado, cada participante era conduzida a uma sala silenciosa onde permanecia com a presença apenas da pesquisadora; então, realizava-se a aplicação do teste Miniexame do Estado Mental, no qual eram utilizados como recursos auxiliares uma folha de papel em branco do tipo Chamex A4, uma caneta esferográfica da marca Bic e um relógio de pulso. Em seguida realizava-se a aplicação da Escala de Epworth para avaliação da qualidade do sono. Após a aplicação destes, foram fornecidos às participantes os valores das pontuações alcançadas em ambos os testes, com suas respectivas interpretações, além de se sanarem dúvidas referentes aos resultados obtidos. 0 tempo despendido em cada avaliação foi de cerca de 20 minutos.

\section{Grupos experimentais}

As 60 participantes do estudo foram divididas de acordo com o tempo que praticavam exercício:

- Sedentárias: grupo composto por 20 mulheres que não praticavam exercício físico.

- Praticantes de exercício físico há um ano: grupo composto por 20 mulheres que realizavam a prática regular de atividade física por um período ininterrupto de até um ano, com frequência igual ou superior a três vezes por semana com carga horária diária de 60 minutos.

- Praticantes de exercício físico por mais de dois anos: grupo composto por 20 mulheres que realizavam a prática regular de atividade física por um período ininterrupto e superior a dois anos, também com frequência igual ou superior a três vezes por semana com carga horária diária de 60 minutos.

Os praticantes de exercícios físicos ainda foram subdivididos em grupos conforme o tipo de exercício: os que realizavam somente ginástica e os que realizavam ginástica e caminhada.

1GI - participantes que praticavam somente ginástica por até um ano.

1GICA - participantes que praticavam ginástica e caminhada por até um ano.

2GI - participantes que praticavam somente ginástica por mais de dois anos.

2GICA - participantes que praticavam ginástica e caminhada por mais de dois anos. 


\section{Análise estatística}

Os dados foram avaliados quanto ao coeficiente de variação e à distribuição amostral para determinação do teste estatístico considerando o nível de significância estatística de $5 \%(p<0,05)$. Empregou-se o teste de Wilcoxon para realização dessa análise.

\section{Resultados}

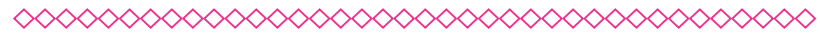

No presente estudo, foram realizadas amostras com 60 mulheres, donas de casa com faixa etária de 50 a 75 anos, e, no grupo composto por 20 mulheres sedentárias, a idade variava entre 50 a 70 anos, com média de idade de 62,41 . No grupo praticante de exercícios físicos até um ano, a faixa etária compreendia de 52 a 75 anos, com média de idade de 68,93 anos. Já no conjunto das 20 muIheres que praticavam exercícios físicos há mais de dois anos, a faixa etária compreendia de 53 a 72 anos, com média de idade de 64,75 anos.

$\mathrm{Na}$ análise intergrupos, os resultados obtidos do Miniexame do Estado Mental entre sedentários versus um ano de exercício, houve diferença estatisticamente considerável $(p<0,0005)$, o mesmo ocorreu para o grupo com um ano de exercício vs. grupo com dois anos de prática de exercícios $(p<0,007)$. Dos grupos sedentários vs. mais de dois anos de exercícios, o resultado foi $p<0,05$ (Tabela 1).

$\mathrm{Na}$ análise intergrupos, os resultados obtidos na Escala de Epworth entre os grupos sedentários vs. um ano de exercício, grupo um ano de exercício físico vs. grupo dois anos de prática de exercício físico e grupo sedentários vs. dois anos de exercícios, houve diferença estatisticamente significativa com $p<0,0002, p<0,0007$ e $p<0,05$ respectivamente (Tabela $\mathbf{1}$ ).

Na comparação no quesito modalidade de atividade física praticada em relação ao desempenho do Miniexame do Estado Mental (Tabela 2), não foram observadas diferenças estatisticamente significativas entre o grupo 1 GI vs. 1GICA, $(p<0,93)$. 0 mesmo ocorreu entre os grupos 1GI vs. 2GI $(p<0,21)$ e para o grupo 1GICA vs. 2 GI $(p<0,18)$. Também não houve diferenças estatisticamente significativas $(p<0,48)$ entre o grupo que 2GICA vs. 2 GI. Nos grupos 1 GI vs. 2 GICA e 1 GICA vs. 2 GICA, ocorreram diferenças estatísticas significativas com $p<0,02$ e $p<0,01$ respectivamente.

$\mathrm{Na}$ comparação no quesito modalidade de atividade física praticada em relação ao desempenho da Escala de Epworth (Tabela 3), foi observado que não houve diferença estatística significativa para os indivíduos do 1GI vs. 1GICA $(p<0,93)$. 0 mesmo ocorreu entre os grupos 2GI vs. 2 GICA $(p<0,11)$. Também não houve diferenças estatisticamente significativas entre o grupo $1 \mathrm{GI}$ vs. $2 \mathrm{GI}(\mathrm{p}<0,09)$. Nos grupos, 1GI vs. 2GICA $(p<0,01), 1$ GICA vs. 2GICA $(p<0,004)$ e 2GICA vs. 2GI $(p<0,02)$, observaram-se diferenças estatísticas significativas.

\section{Discussão}

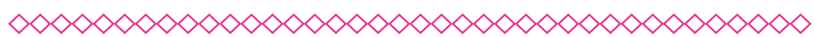

Observou-se que o grupo formado de mulheres sedentárias apresentou o menor escore no Miniexame do Estado Mental quando comparado com os participantes que praticavam atividade física. Esse resultado vai de acordo com Chiari et al. ${ }^{7}$, que afirma que a falta de atividade física promove o avanço mais rápido do processo de degeneração cerebral, o que reflete diretamente na perda da capacidade cognitiva e interfere no processo de formação e evocação da memória e da capacidade de raciocínio lógico. Mesmo com um escore considerado baixo em relação às demais participantes, esse valor ainda se encontra dentro da faixa de normalidade de acordo com Inouye ${ }^{8}$, que afirma que valores iguais ou superiores a 18 são adequados para pessoas com escolaridade no nível de Ensino Fundamental, o que corrobora com os achados deste estudo, haja vista que os indivíduos que obtiveram a menor nota também apresentaram baixa escolaridade. Esses achados refletem diretamente na importância de manter-se ativo na velhice, para que a senilidade possa ocorrer de forma saudável e que a mente e o corpo possam permanecer em sintonia, já que a inatividade por desuso segundo Fechine e Trompieri ${ }^{4}$, gera uma potencialização de alterações tanto estruturais quanto neuroquímicas, 0 que favorece o declínio Sensório-motor e cognitivo do indivíduo em decorrência do envelhecimento. 
Antunes et al. ${ }^{9}$ complementa destacando que a prática regular de um programa de exercícios físicos de intensidade moderada promove uma melhoria no fluxo sanguíneo cerebral, induzindo a angiogênese no tecido cerebral, o que gera uma melhor oxigenação e nutrição do tecido nervoso, mantendo-o mais ativo e funcional com preservação de maior número de conexões, garantindo, assim, a manutenção da memória de forma eficiente. Vorkapic ${ }^{10}$ explica que o aumento do fluxo sanguíneo gerado pelo exercício causa um aumento da produção de endorfina e melhora a capitação de lactato e glicose, que atuam como importantes combustíveis cerebrais e potencializam as funções cognitivas no córtex.

0 grupo formado por mulheres praticantes de atividades físicas há mais de dois anos apresentou o melhor escore no Miniexame do Estado Mental dentre os três grupos (Tabela 1). Esses dados são semelhantes aos estudos de Schaeffer et al. ${ }^{11}$, que observou, em seu estudo, que os idosos fisicamente ativos submetidos a um teste de memória episódica, de longo prazo, apresentaram desempenho significativamente melhor do que o grupo inativo. Esses dados sugerem que o tempo de prática de exercícios está diretamente relacionado com melhores desempenhos cognitivos, e que a prática deste já demonstra bons resultados para a saúde mental.

Dias et al. ${ }^{12}$ complementa este resultado quando explica que o indivíduo que se exercita durante toda a sua vida apresenta um grande potencial de formação de novos neurônios no hipocampo mas, especificamente no giro denteado, que é uma região importante para a formação de novas memórias, além de fortalecer e estimula novas sinapses nos neurônios já existentes. Essa capacidade de deflagrar mudanças na atividade neural decorrentes da prática de atividade física em longo prazo, promove estimulação da plasticidade neural, quando o exercício gera um aumento da atividade da enzima mitocondrial citocromo oxidase (COX) em determinadas estruturas cerebrais, atuando no transporte de elétrons, na produção de ATP, que é fonte de energia celular, e no combate aos radicais livres, bem como agindo como protetor cerebral ${ }^{10}$.

Tanto as mulheres do grupo que realizava atividade física há um ano quanto as do grupo que realizava exercícios há mais de dois anos, além de melhores desempenhos no Miniexame do Estado Mental, apresentaram-se para a avaliação mais comunicativas, mais bem-humoradas que as sedentárias, em sua maioria. Esse fato está de acordo com Chiari et al. ${ }^{7}$ e ocorre pelo melhor convivio social, pela promoção do bem-estar, pela melhoria do humor e pela redução do risco de possível demência que a prática de atividade física promove.

0 grupo formado por mulheres sedentárias apresentou o maior escore, com diferença estatística significativa, na Escala de Epwoprth (Tabela 1), quando comparado com as mulheres que praticavam atividade física, indicando um excesso de sonolência diurna nas mulheres sedentárias, devido à má qualidade de sono noturno. Back et al. ${ }^{13}$ relata que, à medida que o ser humano envelhece, ocorre uma fragmentação do ciclo sono-vigília, levando o idoso a acordar diversas vezes durante a noite e a realizar cochilos durante o dia na tentativa de suprir o tempo de sono incompleto. Esse fator é mais acentuado quando há carência da prática de atividades físicas, já que estas estimulam e exercem influência em todos os sistemas corpóreos, inclusive no estado de sono e vigília.

Segundo Wang e Yongstedt ${ }^{14}$, os mecanismos pelos quais o exercício melhora a qualidade do sono provavelmente são multifatoriais. Tem sido sugerido que os efeitos do exercício sobre o sono estão relacionados com efeitos antidepressivos, redução da ansiedade e alterações nos níveis de serotonina. Powers e Howley ${ }^{15}$ complementam, explicando que a atividade física praticada por longo período de tempo facilita a manutenção de um bom padrão de sono, pois reduz a reserva energética corpórea, aumentando a necessidade de sono, para que, durante esse período, seja estabelecido um balanço energético positivo, o qual garantirá uma boa taxa energética para que o indivíduo permaneça alerta durante a vigília.

0 grupo que realizava apenas ginástica até um ano não apresentou diferença estatística significativa no Miniexame do Estado Mental quando comparado ao grupo que praticava ginástica e caminhada no mesmo período de tempo. 0 mesmo ocorreu no grupo que praticava somente ginástica há mais de dois quando comparado ao grupo que praticava ginástica e caminhada há mais de dois 
anos (Tabela 2). Os idosos que praticam atividade física regularmente, independentemente do tipo de atividade, mantêm o corpo em boas condições físicas, trazendo benefícios também à memória de idosos saudáveis ou portadores de condições patológicas que acometem o sistema cognitivo. Pesquisas demonstram que esses benefícios na memória podem estar associados aos efeitos do exercício físico sobre o sistema cardiovascular, pois há aumento da circulação sanguínea no cérebro, consequentemente aumentando a oxigenação ${ }^{16,17}$.

Em relação ao tipo de exercício realizado e a escala de Epworth, foi observada diferença estatística significativa entre $1 \mathrm{GI}$ vs. 2GICA, 1GICA vs. 2GICA e 1GICA vs. 2GI, indicando que o grupo que praticava algum tipo de exercício por até um ano apresentou uma pior qualidade do sono quando comparado ao das mulheres que praticavam algum tipo de exercício por mais de dois anos. O sono apresenta características comportamentais em relação ao exercício, onde o tempo total de sono mostra uma relação linear com a duração do exercício, e os maiores aumentos no tempo total de sono foram observados após exercícios de maiores durações ${ }^{18}$. Atkinson e Davenne ${ }^{19}$ destacam, ainda, que o horário em que o exercício é realizado é fundamental na indução de melhorias no padrão de sono, visto que exercícios realizados pela manhã são benéficos, podem melhorar o sono; porém, se praticados no fim da tarde e noite, podem surtir o efeito contrário, independentemente da modalidade praticada.

\section{Conclusão}

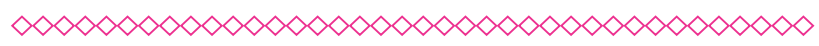

Mediante os resultados deste estudo, sugere-se que ocorreu a melhoria da qualidade de sono e da memória em idosas que praticavam atividade física. Observou-se que o idoso ativo apresenta melhores desempenhos tanto físicos como cognitivos, assim como uma melhoria no padrão do sono em relação aos sedentários de mesma faixa etária. Porém, mais estudos sobre este tema são necessários.

\section{Referências}

$\infty<\infty<\infty<\infty<\infty<\infty<\infty<\infty<\infty<\infty<\infty<\infty<\infty<\infty<\infty<\infty$

1. Mendes MR, Gusmão JLD, Faro ACM, Leite RDCBD. A situação social do idoso no Brasil: uma breve consideração. Acta paulista de enfermagem. 2005; 18(4):422-26.

2. Oliveira BHD, Yassuda MS, Cupertino APFB, Neri AL. Relações entre padrão do sono, saúde percebida e variáveis socioeconômicas em uma amostra de idosos residentes na comunidade: Estudo PENSA. Ciência \& Saúde Coletiva. 2010; 15(3):851-60.

3. Santos AAD, Mansano-Schlosser TCDS, Ceolim MF, Pavarini SCI. Sono, fragilidade e cognição: estudo multicêntrico com idosos brasileiros. Revista Brasileira de Enfermagem. 2013 maio-jun; 66(3):351-357.

4. Fechine BRA, Trompieri N. O processo de envelhecimento: as principais alterações que acontecem com o idoso com o passar dos anos. Inter Science Place. 2015; 1(20):106-94.

5. Bourscheid FR, Mothes L, Irigaray TQ. Memória em idoso: relação entre percepção subjetiva e desempenho em testes objetivos. Estud. psicol. 2016; 33(1):151-59.

6. Onen F, Moreau T, Gooneratne NS, Petit C, Falissard B, Onen SH. Limits of the Epworth Sleepiness Scale in older adults. Sleep and Breathing. 2013; 17(1):343-50.

7. Chiari H, de Mello MT, Rezeak P, Antunes HKM. Exercício físico, atividade física e os benefícios sobre a memória de idosos. Revista Psicologia e Saúde. 2010; 2(1):42-49.

8. Inouye SK. Aspectos neuropsiquiátricos do envelhecimento. In: Goldman, L. Ausiello, D. Cecil - Tratado de Medicina Interna. 23. ed. São Paulo: Elsevier; 2009. p. 160-64.

9. Antunes HK, Santos RF, Cassilhas R, Santos RV, Bueno OF, Mello MTD. Exercício físico e função cognitiva: uma revisão. Revista Brasileira de Medicina do Esporte. 2006; 12(2):108-14.

10. Vorkapic CF. Cérebro em Forma. Revista Viver Mente e Cérebro. 2007; 2(170):74-81. 
11. Schaeffer EL, Zaninotto ALC, Miotto EC, Lucia MCS, Scaff M. Avaliação das funções mnésticas e atencionais em uma amostra de idosos saudáveis engajados em atividade física regular. Psicol. hosp. 2011; 9(1):97-115.

12. Dias RG, Streit IA, Sandreschi PF, Benedetti TRB, Mazo GZ. Diferenças nos aspectos cognitivos entre idosos praticantes e não praticantes de exercício físico. J Bras Psiquiatr. 2014; 63(4):326331.

13. Back FA, Fortes FS, Santos EHR, Tambelli R, Menna-Barreto LS, Louzada FM. Sincronização não-fótica: o efeito do exercício físico aeróbio. Rev Bras Med Esporte. 2007; 13(2):138-42.

14. Wang, Xuewen; Youngstedt, Shawn D. Sleep quality improved following a single session of moderate-intensity aerobic exercise in older women: Results from a pilot study. Journal of sport and health science. 2014; 3(4):338-42.

15. Powers SK, Howley ET. Fisiologia do exercício: teoria e aplicação ao condicionamento e ao desempenho. 8. ed. São Paulo: Manole; 2014. p. 521.

16. Silva NL, Brasil C, Furtado H, Costa J, Farinatti P. Exercício Físico E Envelhecimento: Benefícios À Saúde e Características De Programas Desenvolvidos Pelo Labsau/Iefd. Uerj. Rev. Hupe. 2014; 13(2):75-85.

17. Dias MSL, Moreno R. Estimulação cognitiva por meio de atividades físicas em idosas: examinando uma proposta de intervenção. Rev Bras Geriatr Gerontol. 2012; 15(2):325-334.

18. Reid KJ, Baron KG, Lu B, Naylor E, Wolfe L, Zee PC. Aerobic exercise improves self-reported sleep and quality of life in older adults with insomnia. Sleep medicine. 2010; 11(9):934-40.

19. Atkinson G, Davenne D. Relationships between sleep, physical activity and human health. Physiology \& behavior. 2007; 90(2):229-35. 


\section{Anexos}

Tabela 1 - Relação do teste de Minimental e Escala de Epworth.

\section{Memória (minimental) e sono (Epworth)}

$\begin{array}{llll}\text { Testes } & \text { Sedentário } & \text { Até } 1 \text { ano de exercício } & \text { Mais de } 2 \text { anos de exercício } \\ \text { Minimental } & 22,05 \pm 0,93^{\mathrm{a}, \mathrm{b}} & 24,25 \pm 0,90^{\mathrm{a}, \mathrm{c}} & 25,9 \pm 0,93^{\mathrm{b}, \mathrm{c}} \\ \text { Epworth } & 13 \pm 1,46^{\mathrm{a}, \mathrm{b}} & 9,5 \pm 1,49^{\mathrm{a}, \mathrm{c}} & 5,65 \pm 1,52^{\mathrm{b}, \mathrm{c}}\end{array}$

Dados expressos em média e erro padrão. Resultado considerando significativamente quando $p<0,05$ (a- SED vs. 1EXER, b- SED vs. 2EXER, c- 1EXER vs. 2EXER).

(clique para voltar ao texto)

Tabela 2 - Escore do teste Minimental por modalidade de atividade física.

\section{Minimental}

\section{Tipo de Exercício Físico}

\section{Ginástica}

Ginástica + caminhada
Até 1 ano de exercício

$$
24,65 \pm 1,03^{c}
$$

$24 \pm 0,82^{\mathrm{d}}$
Mais de 2 anos de exercício $25,85 \pm 0,78$ $25,92 \pm 1,02^{c, d}$

Dados expressos em média e erro padrão. Resultado considerando significativamente quando $p<0,05$ (a- 1GI vs. 1GICA por b- 1 GI vs. 2GI, c- 1GI vs. 2GICA, d- 1GICA vs. 2GICA, e- 1GICA vs. 2GI, f- 2GI vs. 2GICA).

(clique para voltar ao texto)

Tabela 3 - Escore da Escala de Epworth por modalidade de atividade física.

\section{Escala de Epworth}

\section{Tipo de Exercício Físico}

\section{Ginástica}

Ginástica + caminhada

\section{Até 1 ano de exercício}

$8 \pm 0,84^{c}$

$10 \pm 1,81^{\mathrm{d}, \mathrm{e}}$

\section{Mais de 2 anos de exercício}

$5,57 \pm 1,52^{c, e}$

$5,69 \pm 1,57^{c, d}$

Dados expressos em média e erro padrão. Resultado considerando significativamente quando $p<0,05$ (a- 1 GI vs. 1GICA por b- 1 GI vs. 2GI, c- 1GI vs. 2GICA, d- 1GICA vs. 2GICA, e- 1GICA vs. 2GI, f- 2GI vs. 2GICA). 